\title{
Kawada, Junzo. - Genèse et dynamique de la royauté : les Mosi méridionaux (Burkina Faso)
}

Préface de Georges Balandier. Paris, L'Harmattan («Études africaines »), 2002, 396 p.

\section{Yveline Dévérin}

\section{CpenEdition}

\section{Journals}

Édition électronique

URL : http://journals.openedition.org/etudesafricaines/5899

DOI : 10.4000 /etudesafricaines.5899

ISSN : $1777-5353$

Éditeur

Éditions de l'EHESS

\section{Édition imprimée}

Date de publication : 31 mars 2006

Pagination : 219-224

ISBN : 978-2-7132-2089-0

ISSN : 0008-0055

\section{Référence électronique}

Yveline Dévérin, «Kawada, Junzo. - Genèse et dynamique de la royauté : les Mosi méridionaux (Burkina Faso) », Cahiers d'études africaines [En ligne], 181 | 2006, mis en ligne le 13 avril 2006, consulté le 23 septembre 2020. URL : http://journals.openedition.org/etudesafricaines/5899 ; DOI : https://doi.org/ 10.4000/etudesafricaines.5899

Ce document a été généré automatiquement le 23 septembre 2020

(c) Cahiers d'Études africaines 


\section{Kawada, Junzo. - Genèse et dynamique de la royauté : les Mosi méridionaux (Burkina Faso)}

Préface de Georges Balandier. Paris, L'Harmattan (« Études africaines »), 2002, 396 p.

Yveline Dévérin

1 Les éditions L'Harmattan permettent enfin à tous ceux qui s'intéressent aux royaumes mosi ${ }^{1}$ d'accéder au magistral travail de référence qu'est la thèse de Junzo Kawada soutenue en 1971 et diffusée (mais de façon confidentielle) en 1979 grâce à l'Institut de recherche sur les cultures et les langues d'Asie et d'Afrique (Tokyo). Jamais republié, disparu des centres de documentation et bibliothèques, les jeunes chercheurs ne connaissaient cet ouvrage que par bribes, citations de deuxième, voire de troisième main, au sein d'ouvrages plus anciens. Comme pour se faire pardonner d'avoir tant tardé à nous faire partager ce travail, l'auteur a ajouté à la présente édition cinq articles plus tardifs (de 1977 à 2001) qui viennent compléter certains aspects de la thèse, soulignant la continuité et la méticulosité des recherches poursuivies.

2 Il est tout à fait réconfortant qu'un éditeur à large diffusion permette enfin un accès important à ce travail de référence; on regrettera, toutefois, pour cet ouvrage particulièrement dense, la présentation ramassée (économique ?) qui ne permet pas de souligner la démarche de l'auteur dont les numérotations de paragraphes disparaissent à l'intérieur du texte. Pour une publication qui n'est pas réservée aux spécialistes des royaumes mosi, une carte globale en ouverture, permettant de localiser les différents royaumes évoqués: méridionaux (Tenkodogo), centraux (Ouagadougou), septentrionaux (Yatenga ${ }^{2}$ ) et Manprusi (Ghana), aurait été la bienvenue, ainsi qu'un index et un lexique dont l'absence limite l'efficacité de l'ouvrage.

Genèse et dynamique de la royauté s'annonce comme une étude centrée sur les royaumes méridionaux (Tenkodogo) mais, en fait, l'étude, de par ses importants aspects comparatifs, apporte aussi beaucoup d'informations et d'analyses sur les autres royaumes, excepté le Yatenga ${ }^{3}$. Cet ouvrage ne doit donc en aucun cas être réservé à 
ceux qui travaillent sur les Mosi méridionaux, tant les informations apportées sur le royaume de Ouagadougou et le Manprusi sont nombreuses et fournies. L'auteur va bien au-delà de la réflexion annoncée sur la dynamique de la royauté et de l'organisation d'un État segmentaire: le pouvoir, les rites qui lui sont liés, l'économique et la segmentation de l'État forment l'armature de l'ouvrage.

Outre les informations issues d'enquêtes et de lectures minutieuses, l'ouvrage apporte une réflexion poussée sur les apports et les limites de la tradition orale (usage des tambours compris). En cela, il est précieux pour tous ceux qui travaillent sur la tradition orale, dépassant ainsi le public africaniste. Il pose particulièrement bien le problème de l'influence coloniale et montre la difficulté à faire la part des éléments purement précoloniaux de ceux qui, hérités de la période précoloniale, ont été ensuite transformés : nous sommes ici dans le cadre d'une histoire racontée/écrite a posteriori qui porte donc l'empreinte non négligeable de la période de narration/écriture. Junzo Kawada souligne ainsi, au sein de sa thèse et dans un des articles postérieurs, l'importance du langage tambourinaire dont il démontre qu'il présente l'avantage d'être moins sujet à déformation que le langage oral.

Une analyse rigoureuse et systématique des terminologies en moore ${ }^{4}$ des thèmes étudiés ${ }^{5}$ tout au long de l'ouvrage prend soin de relever les variantes de sens en fonction des lieux et des périodes, et ces nuances sont précieuses pour le chercheur (pour exemple, les différents types de basga, un rite lié à la chefferie qui est étudié dans ses variantes géographiques et historiques). Enfin, l'approche même est très originale pour le lecteur français: japonais, l'auteur n'a pas été pris dans les dilemmes " postcoloniaux ». Il a un regard tout à fait extérieur qui lui permet de voir les rivalités entre puissances coloniales là où nous sommes plus habitués à poser les problèmes sous l'angle des rapports entre colonisés et colonisateurs. Ainsi, l'épisode sanglant de la colonne Voulet est avant tout présenté comme la marque de la rivalité avec l'Angleterre (annulation du traité signé en 1894 entre Naaba Wobgo - royaume de Ouagadougou - et Fergusson) ${ }^{6}$. Tout en effaçant les disparités d'approche liées au partage colonial, l'auteur montre comment s'est opérée la séparation culturelle Manprusi/Mosi liée à la colonisation par deux puissances différentes. Il met en valeur le foyer original de Gambaga (Manprusi), trop souvent considéré comme un simple épisode d'une légende fondatrice, rétablissant dès lors la relation historique que l'arbitraire colonial a rompue ${ }^{7}$. Les sociétés mosi, manprusi et dagomba n'avaient jusqu'à présent jamais été étudiées comme un ensemble, mais séparément, de part et d'autre de la frontière, par des chercheurs aux méthodes différentes et aux perspectives distinctes. La mise en place de la frontière artificielle avait eu pour première conséquence une évolution différente de part et d'autre du $11^{\mathrm{e}}$ parallèle, tant pour les rites que pour l'organisation même des royaumes. Ainsi, dans le cas du royaume de Ouagadougou, par le biais de l'impôt, la colonisation française a amplifié la centralisation, tout en faisant des chefs locaux des relais administratifs ${ }^{8}$.

6 Enfin, tout au long de l'ouvrage, l'auteur croise les sources d'information et opère une synthèse critique. Sa présentation est toujours relative: par exemple, travaillant à partir de légendes, il insiste sur les éléments que l'on retrouve dans les différentes variantes, en fonction des lieux, ou, au contraire, sur les aspects exceptionnels, propres à telle version récoltée à endroit précis.

7 Les quatre premiers chapitres sont consacrés aux questions de méthode («Problèmes de recherche sur l'histoire dynastique») et aux problèmes d'élaboration de savoirs 
événementiels, toujours abordés, non seulement en tant que repères, mais aussi en fonction de ce qu'ils peuvent apporter dans la réflexion historique ("Mise en place des Mosi méridionaux ", "Essai de synthèse des chronologies dynastiques ", « Remarques sur l'histoire des Mosi méridionaux»). Les chapitres suivants sont consacrés aux aspects structurels de fond : "Systèmes de domination et de transmission du pouvoir ", "Fondements économiques », " Notion d'État segmentaire ». Le chapitre "Remarques sur les rites des chefs " peut être classé dans cette catégorie, car son objectif est de montrer l'interprétation politique de ces rites.

8 L'étude politique est largement fondée sur le vocabulaire. J. Kawada pose un regard critique sur les terminologies et leur transcription plus ou moins heureuse en langue française. Il nuance les utilisations de mêmes termes en moore en fonction des différents royaumes et insiste en particulier sur les différences de sens entre Yatenga et Mosi méridionaux. Cette rigueur est précieuse pour toute étude; elle permet de souligner l'unité et la variété des cultures mosi, particulièrement en termes d'organisation politique et de mode de succession. S'appuyant tant sur la terminologie que sur la tradition orale (légendes comprises), il montre l'importance de la « segmentation spatiale » en termes d'organisation politique et d'extension territoriale. Par exemple, l'étude de la légende fondatrice de Riale et Yennenga aboutit à un schéma synthétique prenant en compte les différentes variantes, puis à une modélisation de la segmentation spatiale dynastique qui permet de souligner les récurrences (pp. 139-140).

9 Le chapitre sur les fondements économiques donne des précisions sur l'organisation de la cour et de l'économie. À partir d'une étude centrée sur le royaume de Tenkodogo, il met en relation la pérennité des royaumes appuyée par l'économie (la déception des premiers Européens arrivant dans le Mosi ${ }^{9}$ serait expliquée par le déclin des royaumes). Il montre comment le pouvoir politique s'appuie sur des systèmes de circulation des personnes qui permettent au chef d'avoir de la main-d'œuvre mais avec une contrepartie de redistribution (de produits alimentaires en particulier). L'écart économique entre les familles dépend toutefois de l'accès à la main-d'œuvre. Le principe d'un économique non cumulatif est retenu en conclusion, mais à aucun moment l'auteur ne remet en cause l'économique en termes de valeur associée au pouvoir ${ }^{10}$.

10 Les rites des chefs sont étudiés aux différents niveaux de la hiérarchie concernés. Leur signification, leur déroulement, leur périodicité sont bien entendu précisés, mais l'auteur en analyse aussi les variantes, montrant ainsi l'unité et la diversité culturelle des royaumes. Il utilise, par ailleurs, les points communs et les différences avec d'autres rites coutumiers pour en tirer une interprétation de fonctionnement du pouvoir. Enfin, il souligne les liens diffus avec l'islam et l'originalité de Tenkodogo en termes de rituels.

Le dernier chapitre et la conclusion développent le principe de l'État segmentaire dont l'idée est le fil conducteur de l'ensemble de l'ouvrage. L'originalité de Tenkodogo apparaît alors liée, en grande partie, à la faiblesse des liens de parenté entre chef central et chefs locaux, à la non-assimilation des populations autochtones lors de la conquête (soit chassées, soit assimilées tardivement ${ }^{11}$ ). "Le caractère relativement durable des systèmes politiques mosi, accompagné d'une faible centralisation du pouvoir [territorial ${ }^{12}$ ], pourrait être considéré comme un aspect de la flexibilité due à la permanence des oppositions complémentaires des groupes aux systèmes segmentaires. En même temps, il faudrait poser le problème de la taille maximum de l'assise 
territoriale d'un tel système. Sans écriture et sans mode de communication efficace [...], sans appareil bureaucratique développé, un système politique basé sur les lignages segmentaires des dominants connaîtra nécessairement des scissions inhérentes à la structure politique même, lorsque l'assise [...] dépassera sa taille optimale " (pp. 229-230).

Les articles postérieurs à la thèse de l'auteur viennent la compléter. «Segmentation et hiérarchie » (1977) ${ }^{13}$ est une réflexion sur la notion segmentaire ne désignant plus un système politique spécifique mais plutôt un aspect du phénomène politique. L'étude porte sur les types de segmentation, en fonction des groupes et suivant les royaumes. Elle pose le problème du rapport entre les types de segmentation et la hiérarchisation au sein du groupe dominant et souligne les liens avec les populations autochtones. La conclusion reprend les idées développées dans les dernières pages de la thèse. Le «Panégyrique royal tambouriné » (1983) ${ }^{14}$ décrit les messages diffusés par les tambours en tant que «dispositif étatique de communication de masse et de contrôle idéologique" (p.311). L'accent est mis sur le bendre (tambour formé d'une grosse calebasse); l'étude porte sur la communication sonore, autant du point de vue spectrographique que symbolique ou pratique. L'auteur souligne la faiblesse des «représentations plastiques» (statuaire, masques) qui contraste avec l'importance du "dispositif sonore». Les rythmes du bendre sont de véritables "blasons sonores", en donnant au terme son sens médiéval ${ }^{15}$. «Histoire orale et imaginaire du passé » (1993) ${ }^{16}$ nous conduit, au travers de l'exemple du kabsgo (récitation de la généalogie dynastique de l'ancien royaume de Tenkodogo) à mesurer la part de l'information de celle de la poésie et de l'histoire réappropriée en fonction des valeurs contemporaines des récitants qui mettent l'accent soit sur l'épique, soit sur la "chronique ». Le problème des rapports entre « Historicité et subjectivité » $(2000)^{17}$ est dès lors posé et développé à propos d'un "passé actualisé en pays mosi ». Réflexion sur la façon dont les coutumes et le passé sont réinventés en fonction des valeurs contemporaines marquées par le " ouagadougoucentrisme ", particulièrement en ce qui concerne les légendes et mythes fondateurs. Les conséquences de ces réinventions, compte tenu de la diversité des royaumes et de l'importance de la hiérarchie, peuvent mener à des quiproquos diplomatiques ${ }^{18}$. L'auteur prône une « dimension éthique du passé ».

La double orientation " épique » ou "chronique » dans la tradition orale est étudiée dans le dernier article "Epic and Chronicle» (2001) ${ }^{19}$. L'auteur s'appuie sur le récit épique de Sunjaata ${ }^{20}$ (Mali), qu'il oppose aux chroniques des rois (sarkii) hausa du Niger et du Nigeria. La majeure partie de l'article se consacre ensuite à l'importance des langages tambourinaires et revient sur le rôle du bendre chez les mosi. Cette fois, l'étude porte sur la fonction langagière du tambour et élabore une véritable "linguistique tambourinaire».

Cet ouvrage très dense est remarquable de par sa très grande précision au niveau de la démarche méthodologique, dans l'inventaire des textes utilisés et des types de sources orales. Il est une véritable somme des savoirs tout en étant une analyse de premier ordre. L'auteur conclut sur la notion d'État segmentaire et des variantes à appliquer à cette notion suivant les royaumes. Il nous offre un véritable travail sur l'unité et la pérennité des royaumes mosi, tout en soulignant les nuances et la diversité interne. Les notes rassemblées à la fin de la thèse et à la fin de chaque article additif sont des références précieuses. De par son approche, il s'adresse à tout chercheur de sciences humaines (historien, géographe, sociologue, ethnologue, anthropologue) s'intéressant 
aux royaumes mosi, mais aussi à tout État segmentaire et à toute autre région de tradition orale, tant l'apport méthodologique est important.

\section{NOTES}

1.. Nous gardons ici l'orthographe retenue par l'auteur (on trouve aussi en français : Mossi, Moose, Mose pour les formes au pluriel; Moaga pour le singulier mais peu utilisé).

2.. Nous gardons l'orthographe courante en français lorsqu'une orthographe s'est imposée.

3.. Mais ce dernier royaume est bien connu grâce aux travaux de M. IZARD. Voir, entre autres, Gens du pouvoir, gens de la terre. Les institutions politiques de l'ancien royaume du Yatenga (bassin de la Volta Blanche), Cambridge, Cambridge University Press ; Paris, Maison des sciences de l'homme, 1985.

4.. Le français écrit aussi «moré ».

5.. En particulier, tout le vocabulaire ayant trait à la succession, au pouvoir, à l'économique, aux rites.

6.. En décembre 1896, la colonne Voulet-Chanoine (mandatée pour arriver avant les Anglais) entre à Ouagadougou et se heurte à Naaba Wobgo, l'empereur des Mosi. Des combats violents conduisent à la destruction partielle du palais, à l'incendie de la ville et à la fuite de Naaba Wobgo, que les Français remplacent par son frère Naaba Sigri, qui leur est très favorable.

7.. Voir la préface de G. BALANDIER dans l'ouvrage.

8.. Toutefois la puissance coloniale commet parfois des erreurs d'appréciation, inversant (involontairement) les hiérarchies en se référant à ses propres critères (importance du « territoire ») et en ne prenant parfois pas en compte les critères des Mosi (place par rapport au Mogho Naaba).

9.. Voir L.-G. BINGER, Du Niger au golfe de Guinée par le pays de Kong et le Mossi (1887-1889), Paris, Hachette, 1892 [ $1^{\text {re éd.]. }}$

10.. M. IZARD a bien montré l'importance du pouvoir sur les hommes, la fonction de redistribution (fondée sur l'économique) n'étant qu'un moyen d'asseoir ce pouvoir. 11.. Alors que, dans les royaumes de Ouagadougou et du Yatenga, les autochtones ont été très tôt assimilés.

12.. Plus haut, l'auteur souligne la faiblesse de la centralisation territoriale.

13.. Voir Journal of Asian and African Studies, 14, 1977, pp. 144-168.

14.. Voir Journal of Asian and African Studies, 26, 1983, pp. 144-168.

15.. On remarquera qu'en 2005 le bendre est toujours présenté comme la bannière sonore de la culture mosi. Il a d'ailleurs fait l'objet d'études spécifiques lancées au début des années 1990 par Frédéric Tintinga PACERE, créateur du musée de la bendrologie de Manega, musée de la Culture mosi. Voir T. F. Pacere, Le langage des tam-tams et des masques en Afrique, Paris, L'Harmattan, 1992.

16.. Voir Annales. Économies, sociétés, civilisations, 4, juillet-août 1993, pp. 1087-1105. 
17.. J. L. JAMARD, E. TERRAY \& M. XANTHAKOU (dir.), En substance, textes pour Françoise Héritier, Paris, Fayard, 2000, pp. 57-75.

18. L'auteur raconte avec saveur un incident lors de la visite à Gambaga (Ghana, Manprusi) du chef de Tenkodogo.

19.. S. SOGNER (ed.), Making Sense of Global History: The $19^{\text {th }}$ International Congress of the Historical Sciences, Oslo, Universitetsforlaget, 2001, pp. 254-264.

20.. Plus connu sous l'orthographe Soundjata. Soundjata Keita, fondateur de l'empire du Mali. 